


\title{
LITOGEOQUÍMICA EN EL DISTRITO MINERO DE ORO DE LLAMAS DE CABRERA (NOROESTE DE ESPAÑA): IMPLICACIONES METALOGENÉTICAS
}

\author{
LITHOGEOCHEMISTRY OF THE LLAMAS DE CABRERA \\ GOLD DISTRICT (NORTHWEST SPAIN): \\ METALLOGENETIC IMPLICATIONS
}

F. Gómez-Fernández ${ }^{1}$, E. Vindel ${ }^{2}$, E. J. González-Clavijo ${ }^{3}$, T. Martín-Crespo ${ }^{4}$ \& V. Sánchez ${ }^{5}$

Resumen - El objetivo de este trabajo ha sido contribuir al conocimiento de la metalogenia de las mineralizaciones hidrotermales de cuarzo-arsenopirita-oro del distrito minero de Llamas de Cabrera, especialmente en relación a la fuente del oro. Para ello se han llevado a cabo una campańa de geoquímica de mineralizaciones y otra más amplia, de litogeoquímica en las rocas encajantes, especialmente en la cuarcita Armoricana y las pizarras de la Formación Luarca. Se han determinado las concentraciones de $\mathrm{S}$, Au, $\mathrm{Ag}, \mathrm{As}, \mathrm{Ba}, \mathrm{Hg}, \mathrm{Sb}, \mathrm{Cd}, \mathrm{Cu}, \mathrm{Mn}, \mathrm{Mo}, \mathrm{Ni}, \mathrm{Pb}$ y Zn en los encajantes y de Au, Ag en las mineralizaciones. Los altos contenidos en $S$ y metales de las pizarras se interpretan como consecuencia de la presencia de sulfuros. Los valores de Au obtenidos en pizarras y en cuarcitas son significativamente más bajos que los citados en la bibliografía. En las cuarcitas sólo se ha detectado Au en las proximidades del yacimiento de El Veneiro, lo que unido a valores significativos de As en las mismas muestras, conduce a interpretar esos valores significativos de oro en relación a una aureola de dispersión hidrotermal ligada al proceso mineralizador. Teniendo en cuenta datos previos, que apuntan a las rocas del entorno geológico como fuente del oro, los bajos contenidos en $\mathrm{Au}$ obtenidos en este

\footnotetext{
${ }^{1}$ Área de Prospección e Investigación Minera. Universidad de León. C/ Jesús Rubio 2, 24004, León, Spain; f.gomez@unileon.es

${ }^{2}$ Departamento de Cristalografía y Mineralogía, Facultad de Ciencias Geológicas, Universidad Complutense, 28040, Madrid, Spain; evindel@geo.ucm.es

${ }^{3}$ IGME. Unidad de Salamanca, C/ Azafranal, 48, 37001, Salamanca, Spain; egc@usal.es

${ }^{4}$ ESCET, E. Departamental II, Universidad Rey Juan Carlos, Móstoles, 28933, Madrid, Spain; tomas. martin@urjc.es

${ }^{5}$ Departamento de Cristalografía y Mineralogía, Facultad de Ciencias Geológicas, Universidad Complutense, 28040, Madrid, Spain; virginia.sanchez@repsol.com
} 
trabajo, tanto en pizarras como en cuarcitas alejadas de las mineralizaciones ( $<2 \mathrm{ppb})$, se interpretan como debidos a una lixiviación masiva de oro de las rocas durante el proceso mineralizador, previamente a su deposición en zonas extensionales.

Palabras-clave - litogeoquímica, yacimientos de oro, minería romana, Macizo Ibérico

\begin{abstract}
This paper is a contribution to the understanding of the metallogeny of the quartz-arsenopyrite-gold deposits in the Llamas de Cabrera mine-district. The major aim is to disclose the source of the gold involved in the hydrothermal processes which generated these deposits. For this purpose a geochemical study of ore deposits and a lithogeochemistry, especially in the Armorican quartzite and slates from Luarca Formation, were carried out. Sulphur, Au, $\mathrm{Ag}, \mathrm{As}, \mathrm{Ba}, \mathrm{Hg}, \mathrm{Sb}, \mathrm{Cd}, \mathrm{Cu}, \mathrm{Mn}, \mathrm{Mo}, \mathrm{Ni}, \mathrm{Pb}$ and $\mathrm{Zn}$ have been determined in host rocks and $A u, A g$ in mineralized bodies. High contents of $S$ and metals from slate are interpreted as related to the presence of sulphides. Au contents in slate and quartzite are significantly lower than those referred in the literature. Exclusively close to the Veneiro ore deposit was detected $A u$ in quartzite. The presence of $A$ s in these samples suggest that gold mineralization is related to deposition in an halo of hydrothermal dispersion. Previous data point to the surrounding rocks as the source of gold. The low Au values obtained in this work -both in slate and quartzite far away from the ore bodies ( $<2 p p b)$ - are also understand as gold was leached from the enclosing geological bodies prior to its deposition in extensional restricted areas.
\end{abstract}

Keywords - lithogeochemistry, gold deposits, roman mining, Iberian Massif

\title{
1 - Introducción
}

El Noroeste del Macizo Ibérico fue uno de los distritos productores de oro más importantes durante época romana, en la que se explotaron dos tipos de mineralizaciones: filones de cuarzo-oro y depósitos aluviales. Los espectaculares trabajos mineros romanos en el Noroeste de la Península Ibérica han sido objeto de varios estudios arqueológicos (SÁNCHEZ-PALENCIA, 1983; PÉREZ-GARCÍA et al., 2000). En este área se han registrado aproximadamente 500 trabajos mineros históricos, con una producción total de oro durante los siglos I y II estimada en torno a $195 \mathrm{t}$, la mayor parte procedente de yacimientos aluviales, con una ley media de $67 \mathrm{mg} \mathrm{Au} / \mathrm{m}^{3}$ y una ley máxima de 1-2 g/ $\mathrm{m}^{3}$. Desde tiempos romanos hasta la actualidad, las minas de oro en el noroeste de España han permanecido inactivas, excepto para pequeńas operaciones a finales del siglo XIX y primera mitad del siglo XX. No obstante, con el incremento de los precios del oro en la década de los años 70, sobrevino una fase de prospección exhaustiva.

El distrito de Llamas de Cabrera aunque fue ampliamente explotado en época romana, permaneció oculto hasta 2002, cuando fue descubierto por MATÍAS \& GÓMEZ-FERNÁNDEZ (2003). En este distrito se pueden ver actualmente importantes trabajos mineros antiguos, minería a cielo abierto, pozos verticales y galerías, con su propia red hidráulica (MATÍAS \& GÓMEZ-FERNÁNDEZ, 2003; GÓMEZ-FERNÁNDEZ et al., 2005).

Recientemente, GÓMEZ-FERNÁNDEZ et al. (2012) han llevado a cabo un exhaustivo estudio del distrito de Llamas de Cabrera en el que: (1) realizan una primera descripción sistemática del yacimiento, (2) caracterizan los fluidos que intervinieron en 
el proceso hidrotermal a partir del estudio de inclusiones fluidas, (3) analizan la variación composicional de la arsenopirita con objeto de usarla como geotermómetro, (4) aportan información sobre las posibles fuentes de los fluidos mineralizadores a partir del estudio de isótopos estables, (5) reconstruyen la evolución de las variables P-T durante las etapas de formación del yacimiento y, (6) contribuyen al progreso del conocimiento sobre este tipo de yacimientos del Noroeste de la Península Ibérica y los comparan con otros semejantes del dominio varisco.

En este marco, el objetivo del presente trabajo es realizar un estudio litogeoquímico que contribuya al conocimiento metalogenético de estas mineralizaciones, especialmente en lo que se refiere a la fuente del oro involucrado en los procesos hidrotermales que generaron estos yacimientos.

\section{2 - Marco geológico}

De acuerdo con la division del Macizo Ibérico propuesta por PÉREZ-ESTAÚN et al. (2004), el area estudiada está situada en la Zona Centroibérica, en el límite con la Zona Asturoccidental-Leonesa. Los yacimientos de oro se sitúan a lo largo del flanco norte del sinclinal de Truchas (Fig. 1), una estructura compleja formada por la interferencia coaxial de las fases 1 y 3 variscas y algunos cabalgamientos de fase 2 vergentes hacia el norte (MARCOS, 1973; PÉREZ-ESTAÚN, 1975; RODRÍGUEZ FERNÁNDEZ et al., 1982).

El registro sedimentario en la región se inicia con la Formación "Ollo de sapo" (Cámbrico superior a Ordovícico inferior), interpretada como un grupo de materiales magmáticos, volcánicos y volcano-sedimentarios (DÍEZ MONTES, 2010). Está cubierta por una espesa sucesión del Ordovícico inferior (MARTÍNEZ CATALÁN et al., 1992) de filitas y cuarcitas que hacia la parte superior se vuelve más rica en el término arena, terminando en una unidad de cuarcita blanca denominada Cuarcita Armoricana. El Ordovícico medio está formado por las pizarras negras de la Formación Luarca. La transición entre la Cuarcita Armóricana y la Fm. Luarca es gradual, y algunos autores han distinguido una serie de transición a nivel local, denominada Formación Rubianes (GUTIÉRREZ MARCO et al., 1999). La secuencia del Ordovícico superior se encuentra discordante sobre la Cuarcita Armoricana y comienza con la Caliza de La Aquiana. El resto del Ordovícico superior ha sido dividido (BARROS LORENZO, 1989) en tres formaciones fundamentalmente detríticas: Casaio, Rozadais y Losadilla.

La Orogenia Varisca, que afectó a todo el Macizo Ibérico, se manifiesta en la región como un proceso tectono-metamórfico polifásico. En el flanco norte del sinclinal de Truchas la única foliación tectónica identificada es de fase 1 varisca, lo que sugiere que las venas mineralizadas son mas jóvenes que esta foliación. Mientras que en el área de estudio no afloran cuerpos magmáticos de ninguna de las fases variscas, hacia el sur y el este (Fig. 1), en el dominio del antiforme del "Sapo de Ollo", afloran diversos granitoides variscos (DÍEZ MONTES, 2010), generados en distintas fases de la orogenia. El metamorfismo regional es de bajo grado (zona de clorita) y es contemporáneo con la fase 1 varisca.

Algunos depósitos contientales, relacionados con la red fluvial, con episodios glaciares cuaternarios y con avalanchas de ladera cubren localmente la región. El contenido en oro de algunos de estos depósitos sedimentarios centró los trabajos mineros durante la Época Romana (HERAIL, 1984; PÉREZ GARCÍA et al., 2000; MATÍAS \& GÓMEZ-FERNÁNDEZ, 2003). 


\section{3 - Las mineralizaciones primarias de oro en el distrito de llamas de cabrera}

El distrito minero de Llamas de Cabrera forma un área extensa en la que se encuen-

tran varios yacimientos primarios de cuarzo-arsenopirita-oro (Fig. 1) encajados en rocas de edad paleozoica. Estas mineralizaciones están estructuralmente controladas y se pueden agrupar en tres tipos: a) filones extensionales de cuarzo, en dirección N-S a N20 $\mathrm{E}, \mathrm{b})$ filones de cuarzo, brechas y stockworks en dirección WNW-ESE, y c) otros yacimientos relacionados con fallas NE-SW. Encajan en las capas superiores de la Cuarcita Armoricana, equivalentes a la Fm. Rubianes, si bien ocasionalmente lo pueden hacer en las pizarras de los tramos inferiores de la Fm. Luarca. Las alteraciones hidrotermales en el encajante incluyen silicificación, cloritización y sericitización. Se reconocen mineralizaciones diseminadas en zonas de alteración hidrotermal el entorno de las mineralizaciones. La paragénesis mineral es bastante simple, fundamentalmente cuarzo-arsenopirita-oro con otros sulfuros muy escasos, tales como esfalerita, galena, calcopirita, pirita, tetraedrita y bismutina. También se desarrolla una fase supergéncia y de oxidación con la presencia de covellina, escorodita, anglesita y goethita.

\section{4 - Metodología}

Para el estudio geoquímico se han llevado a cabo dos tipos de muestreo. El primero ha consistido en una toma de muestras directamente en los yacimientos (Tabla 1; Fig. 1): a) seis muestras puntuales (chip samples) en filones mineralizados (El Veneiro, Pombriego, Pozos, Llamas de Cabrera; Fig. 1), b) una muestra puntual de la salvanda arcillosa que acompaña a uno de estos filones (Llamas de Cabrera) y c) una muestra correspondiente a una roza (channel sample) transversal a la corona de la galería Machato (Llamas de Cabrera). En estas muestras se analizaron los contenidos en $\mathrm{Au}$ y Ag.

El segundo muestreo consistió en una campaña de litogeoquímica extensa en la que se recogieron 25 muestras de roca de caja: Cuarcita Armoricana y Formaciones Luarca y Rozadais. Las litologías muestreadas han sido, cuarcitas, pizarras y alternancias de cuarcitas, analizándose una serie de elementos considerados representativos (Tabla 2). Se tomaron muestras de 5 kilogramos de peso, obtenidas en rozas y en paneles, tanto en zonas alejadas de las mineralizaciones de Au como en sus proximidades (Fig. 1).

Las muestras fueron molidas hasta un tamaño de partícula por debajo de $100 \mu \mathrm{m}$. Los análisis fueron realizados por Activation Laboratories Ltd., en Thunder Bay (Ontario, Canadá). Las metodologías utilizadas fueron ensayo al fuego/gravimétrico ( $\mathrm{Au} \mathrm{y} \mathrm{Ag)} \mathrm{en}$ geoquímica de mineralizaciones, mientras que en geoquímica de rocas se utilizó INAA ( $\mathrm{Au}, \mathrm{As}, \mathrm{Ba}, \mathrm{Hg}, \mathrm{Sb}$ y W), agua regia ICP (Cd, Cu, Mn, Mo, Ni, Pb, Zn y S) e INAA/ agua regia ICP (Ag). Los límites de detección para cada uno de los elementos determinados se encuentran especificados en las Tablas 1 y 2.

\section{5 - Resultados}

Los resultados analíticos de la geoquímica de mineralizaciones de cuarzo-arsenopirita-oro están reflejados en la Tabla 1 . Los valores obtenidos varían entre < 0,03 y 18,7 ppm 
Au. Los contenidos en Ag usualmente quedan por debajo del límite de detección de la técnica instrumental. Dada la metodología utilizada en el muestreo (chip samples), los valores obtenidos, aunque indicativos de la riqueza de los yacimientos, no se pueden considerar representativos de la ley de cada uno de estos, a excepción de la muestra M2 (roza), cuyos valores representan la ley de la galería Machato (Fig. 1), encajada en pizarras de Luarca.

Los resultados analíticos de la litogeoquímica se recogen en la Tabla 2. La metodología del muestreo permite considerar los resultados como representativos de las rocas analizadas. La comparativa entre pizarras y cuarcitas indica que las primeras tienen contenidos significativamente más altos en $\mathrm{S}, \mathrm{Ni}, \mathrm{Pb}, \mathrm{Zn}$ y $\mathrm{Ba}$, y las cuarcitas valores superiores en $\mathrm{Au}, \mathrm{Ag}$, As y W.

\section{6 - Estudios geoquímicos previos}

Aunque no existen muchos datos al respecto, en la bibliografía previa se pueden recopilar algunos datos geoquímicos sobre el contenido en Au de las rocas paleozoicas del Noroeste de la Península Ibérica. Así PORTER \& ÁLVAREZ MORÁN (1992) ofrecen, entre otros, los siguientes valores: a) hasta $80 \mathrm{ppb}$ el contenido de las pizarras de la Fm. Luarca, b) $30 \mathrm{ppb}$ el contenido medio de los materiales vulcanosedimentarios de la Fm. Luarca y c) 4 ppb el contenido medio de las cuarcitas de Los Cabos del oeste de Asturias (JAHODA, 1977).

Por otra parte HERAIL (1984) pone de manifiesto la existencia de paleoplaceres de oro presentes en la Cuarcita Armoricana, concretamente en el área de El Veneiro (Fig. 1). Todo ello ha inducido a pensar que la fuente del oro está en las rocas del entorno geológico (TORNOS et al., 1997), las cuales habrían sido objeto de lixiviación con posterioridad a la primera fase de deformación varisca $\left(\mathrm{D}_{1}\right)$. El transporte del oro habría estado ligado a células convectivas originadas como consecuencia del aumento de temperatura propiciado por el ascenso de intrusivos (Granitoides Variscos postcinemáticos, Fig.1) durante la segunda fase de deformación varisca $\left(\mathrm{D}_{2}\right)$ (GÓMEZ-FERNÁNDEZ et al., 2012). La deposición del oro se produjo en etapas sucesivas a lo largo de fallas extensionales, brechas y stockworks.

Tabla 1 - Resultados analíticos de geoquímica de mineralizaciones

\begin{tabular}{clllccc}
\hline Muestra & Componentes de visu & Tipo & \multicolumn{1}{c}{ Yacimiento } & Encajante & Au (ppm) & Ag (ppm) \\
\hline 24 & Qtz + Goethita & Chip & El Veneiro & Arm. & 0.30 & $<3$ \\
31 & Qtz + Apy + Py & Chip & Pombriego & Arm. & 3.52 & $<3$ \\
PZ & Qtz + Apy & Chip & Pozos & Arm. & 0.50 & $<3$ \\
M31 & Qtz + Apy & Chip & Llamas de Cabrera & Arm. & 18.70 & $<3$ \\
Z5 & Qtz + Apy & Chip & Llamas de Cabrera & Arm. & 3.59 & 7 \\
132B & Qtz + Apy & Chip & Llamas de Cabrera & Arm. & 5.19 & $<3$ \\
M12 & Salvanda arcillosa & Chip & Llamas de Cabrera & Arm. & $<0.03$ & $<3$ \\
M2 & Pizarra + Qtz & Roza & Llamas de Cabrera & Lua. & 2.27 & $<3$ \\
\hline
\end{tabular}

Abreviaturas: Qtz = cuarzo; Apy = arsenopirita; Arm. = Cuarcita Armoricana; Lua. = Formación Luarca 


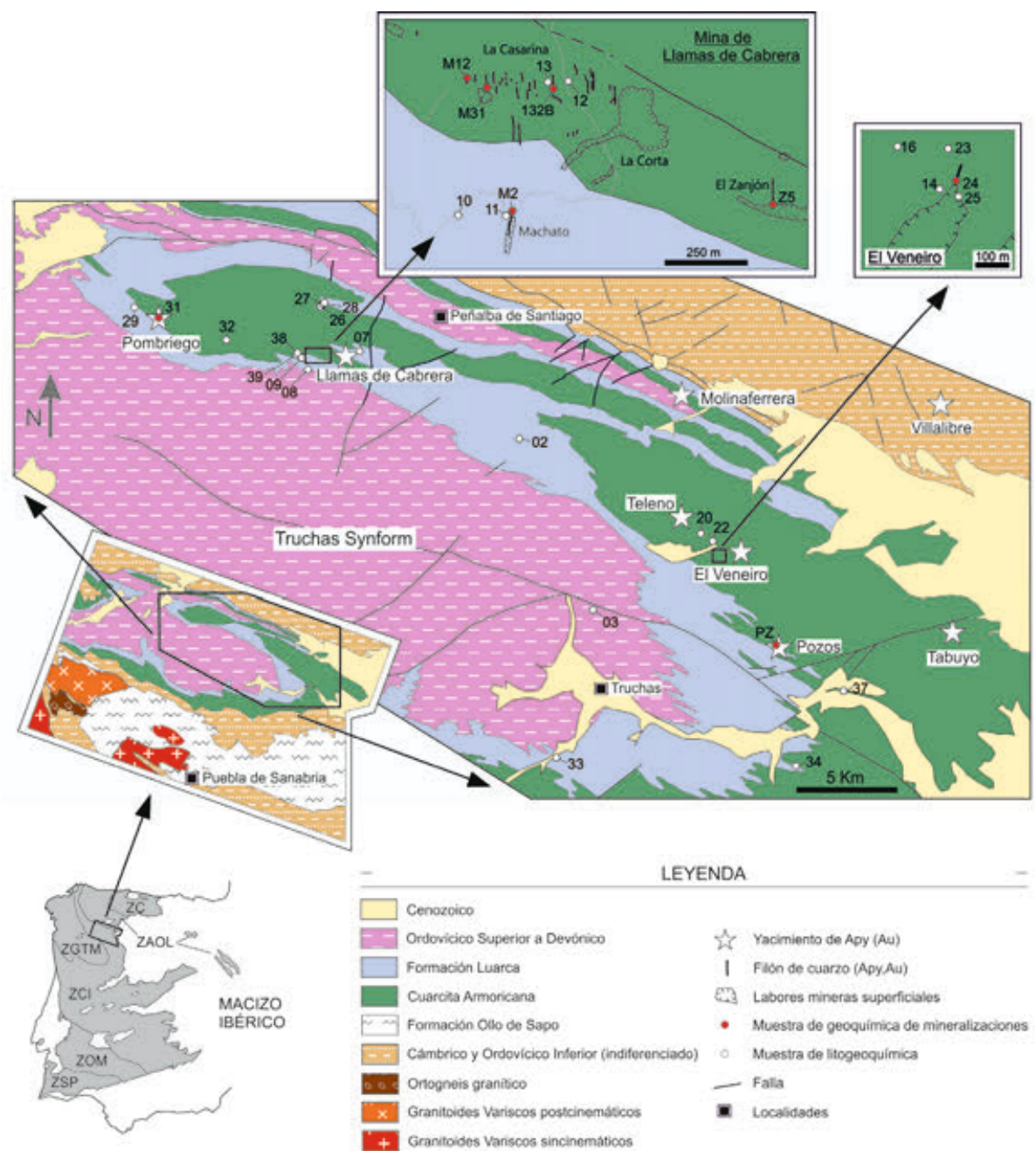

Fig. 1 - Situación de muestras y mapa geológico de la zona de estudio. 


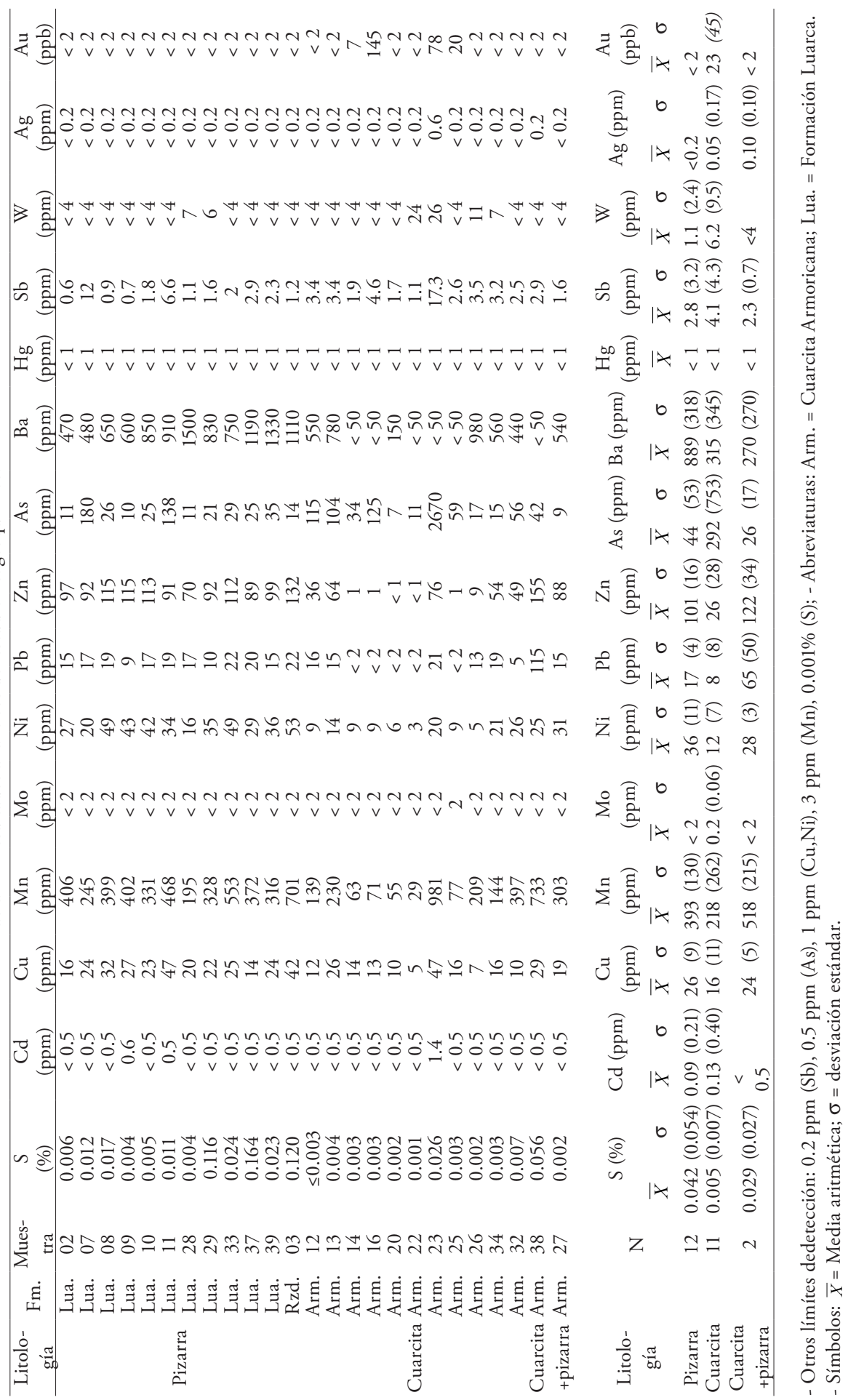




\section{7 - Discusión}

Los altos contenidos en $S$ y metales de las pizarras se interpretan como consecuencia de la presencia de sulfuros, ya indicada por GÓMEZ-FERNÁNDEZ et al. (2009) para pizarras comerciales (pizarras de techar) de esta zona, los cuales fueron precipitados en dos fases de mineralización: a) una fase precinemática, marcada por la deposición en medios sedimentarios anóxicos de sulfuros como pirita, pirrotina, esfalerita, calcopirita, pentlandita, coblatina, ullmanita y gersdorsfita y b) una fase postcinemática, marcada por la precipitación de pirita. Por lo que respecta al origen de los altos contenidos en $\mathrm{Ba}$, las técnicas estrictamente geoquímicas utilizadas en este trabajo no permiten una interpretación sólida, ya que los modos de formación de barita en medios marinos referenciados en la bibliografía son variados, incluyendo mecanismos biológicos y abiológicos (SMITH et al., 2004). Los altos contenidos en W de las cuarcitas probablemente tengan origen sedimentario. La baja solubilidad de wolframita y scheelita hace que estos minerales tiendan a acumularse como detritos de la fracción arena, pudiendo llegar a originar placeres.

Los contenidos en $\mathrm{Au}$, tanto en pizarras como en cuarcitas, merecen un análisis más pormenorizado. Los contenidos de Au en pizarras son significativamente más bajos que los citados por PORTER \& ÁLVAREZ MORÁN (1992), quedando siempre por debajo del límite de detección de la técnica instrumental (2 ppb). En cuanto a las cuarcitas, no se ha detectado Au en rocas alejadas de los yacimientos de cuarzo-arsenopirita-oro. Únicamente se ha detectado en cuatro muestras en el entorno del yacimiento de El Veneiro (muestras 14, 16, 23 y 25; Fig. 1), en las cercanías de una estrecha vena de cuarzo de baja ley de Au. Estas muestras tienen contenidos relativamente altos en As (hasta 2670 ppm, muestra 23).Todo ello sugiere que el oro en la cuarcita podría encontrarse asociado a arsenopirita y consecuentemente no se trataría de oro detrítico, sino relacionado con una aureola de dispersión hidrotermal ligada al proceso mineralizador.

En otras muestras de cuarcita (muestras 12 y 13) recogidas en las proximidades del yacimiento de Llamas de Cabrera, los valores de Au quedan por debajo del límite de detección de la técnica instrumental. Los significativos contenidos en Au de las muestras de estas mineralizaciones (muestras M31, Z5, 132B y M2, Tabla1), comparados con los bajos contenidos de los encajantes, indican que se trataría de un yacimiento de ley superior con una baja dispersión de Au en las rocas del entorno.

\section{8 - Conclusiones}

En GÓMEZ-FERNÁNDEZ et al. (2012) se ha propuesto un modelo mineralizador con implicación de fluidos de origen desconocido ampliamente equilibrados a temperaturas medias-altas con litologías metamórficas, en el que el ascenso de intrusivos durante la segunda fase de deformación varisca $\left(\mathrm{D}_{2}\right)$ actuaría como fuente de calor, motor del desarrollo de células convectivas. En trabajos previos (PORTER \& ÁLVAREZ MORÁN, 1992; TORNOS et al., 1997) se apunta a que la fuente del oro podría estar en las rocas del entorno geológico, hipótesis que concuerda con la presencia de oro detrítico en las cuarcitas, citada por HERAIL (1984). Sin embargo, los datos de geoquímica de rocas que aporta el presente trabajo muestran contenidos en Au incluso inferiores a los referenciados por JAHODA (1977) y PORTER \& ÁLVAREZ MORÁN (1992) para 
las pizarras de Luarca y la Cuarcita Armoricana. La anomalía detectada en esta última unidad estratigráfica no se interpreta como oro detrítico sino como ligado a aureolas de dispersión hidrotermal.

Teniendo en cuenta la idea de que la zona ha sufrido un intenso proceso hidrotermal con una importante circulación de fluidos a una relativa temperatura, la interpretación más razonable para la fuente del oro es que haya sido lixiviado, prácticamente en su totalidad, de rocas encajantes como paso previo a su deposición en zonas extensionales.

Agradecimientos - Este trabajo ha sido financiado por la Excma. Diputación Provincial de León, mediante el proyecto 2005/103 y por la Consejería de Educación de la Junta de Castilla y León, mediante el proyecto LE289A11-2.

\section{Referencias Bibliográficas}

BARROS LORENZO, J.C. (1989) - Nuevos datos geológicos y cartográficos sobre el flanco sur del Sinclinorio de Truchas (Ourense-León, NW de España). Cad. Lab. Xeol. Laxe, 14, p. 93-116.

DÍEZ MONTES, A., MARTÍNEZ CATALÁN, J.R. \& BELLIDO MULAS F. (2010) - Role of the Ollo de Sapo massive felsic volcanism of NW Iberia in the Early Ordovician dynamics of northern Gondwana. Gondwana Res., 17, p. 363-376.

GÓMEZ-FERNÁNDEZ, F, CASTAÑO, M.A., BAULUZ, B. \& WARD, C.R. (2009) - Optical microscope and SEM evaluation of roofing slate fissility and durability. Mater. Construc., 59 (296), p. 91-104.

GÓMEZ-FERNÁNDEZ, F., MATÍAS, R., MÉNDEZ, A.J. y CIFUENTES, J. (2005) - Estudio preliminar de las mineralizaciones de la mina de oro romana de Llamas de Cabrera (León, NO de Espańa). Est. Geol., 61, p. 111-119.

GÓMEZ-FERNÁNDEZ, F., VINDEL, E., MARTÍN-CRESPO, T., SÁNCHEZ V., GONZÁLEZ CLAVIJO, E. \& MATÍAS, R. (2012) - The Llamas de Cabrera gold district, a new discovery in the Variscan basement of northwest Spain: a fluid inclusion and stable isotope study. Ore Geol. Rev. doi: 10.1016/j.oregeorev.2012.02.001

GUTIÉRREZ-MARCO, J.C., ARAMBURU, C., ARBIZU, M., BERNÁRDEZ, E., HACAR RODRÍGUEZ, M.P., MÉNDEZ-BEDIA, I., MONTESINOS LÓPEZ, R., RÁBANO, I., TRUYOLS, J. \& VILLAS, E. (1999) - Revisión bioestratigráfica de las pizarras del Ordovício Medio en el Noroeste de España (Zonas Cantábrica, Asturoccidental-Leonesa y Centroibérica septentrional). Act. Geol. Hisp., 34, p. 3-87.

HERAIL, G. (1984) - Géomorphologie et gîtologie de l'or détritique. Piémonts et bassins intramontagneux du Nord-Ouest de l'Espagne. Centre National de la Recherche Scientifique (CNRS). Paris, 456 p.

JAHODA. R. (1977) - Geology and genesisof auriferous hydromagmatic breccias and related deposits in Northwestern Spain. Unpublished. Ph. D. Univ. Southampton.

MARCOS, A. (1973) - Las series del Paleozoico Inferior y la estructura herciniana del occidente de Asturias (NW de España). Trab. Geol. Univ. Oviedo, 6, p. 1-113.

MARTÍNEZ CATALÁN, J.R., HACAR RODRÍGUEZ, M.P., VILLAR ALONSO, P., PÉREZ ESTAÚN, A. \& GONZÁLEZ LODEIRO, F. (1992) - Lower Paleozoic extensional tectonics in the limit between the West Asturian-Leonese and Central Iberian Zones of the Variscan Fold-Belt in NW Spain. Geol. Rundsch., 81, p. 545-560.

MATIAS, R. y GÓMEZ-FERNÁNDEZ, F. (2003) - La mina romana de Llamas de Cabrera (León-Espańa). Actas del IV Congreso Internacional sobre Patrimonio Geológico y Minero, Utrillas (Teruel), p. 383-398.

PÉREZ-ESTAÚN, A. (1975) - La estratigrafía y la estructura de la rama Sur de la Zona Asturoccidental-Leonesa (W de León, NW de Espańa). PhD Thesis. Universidad de Oviedo, Spain. 
PÉREZ-ESTAÚN, A., BEA, F., BASTIDA, F., MARCOS, A., MARTÍNEZ CATALÁN, J.R., MARTÍNEZ POYATOS, D., ARENAS, R., DÍAZ GARCÍA, F., AZOR, A., SIMANCAS, J.F., \& GONZÁLEZ LODEIRO, F. (2004) - La Cordillera Varisca Europea: el Varisco Ibérico. In: Vera, J.A. (Ed.), Geología de España. SGE-IGME, Madrid, 21-228.

PÉREZ-GARCÍA L.C., SÁNCHEZ-PALENCIA, F.J. \& TORRES-RUIZ, J. (2000) - Tertiary and Quaternary alluvial gold deposits of Northwest Spain and Roman mining (NW of Duero and Bierzo Basins). J. Geochem. Expl., 71, p. 225-240.

PORTER, D.H. \& ÁLVAREZ MORÁN, B. (1992) - Mineralizaciones de oro del noroeste de Espańa. In: García Guinea, J. y Martínez Frías J. (eds.). Recursos Minerales Espańoles. CSIC, Madrid, p. 849-860.

RODRÍGUEZ FERNÁNDEZ, L.R., ABRIL HURTADO, J., PLIEGO DONES, D., \& RUBIO NAVAS, J. (1982) - Mapa Geológico de España E. 1:50.000, Segunda Serie. Servicio Publicaciones del Ministerio de Industria y Energía, Memoria 56 p.

SÁNCHEZ-PALENCIA, F.J. (1983) - La explotación del oro de Asturia y Gallaecia en la Antigüedad, PhD Thesis, Univ. Complutense, Madrid.

SMITH, E., HAMILTON-TAYLOR, J., DAVISON, W., FULLWOOD, N.J. \& MCGRATH, M. (2004) - The effect of humic substances on barite precipitation - dissolution behaviour in natural and synthetic lake waters. Chem. Geol., 207, p. 81-89.

TORNOS, F., SPIRO, B.F., SHEPHERD, T.J. \& RIBERA, F. (1997) - Sandstonehosted gold lodes of the Southern West Asturian Leonese Zone (NW Spain). Chron. Rech. Min., 528, p. 71-86. 